\title{
Atypical COVID-19 Infections - The Challenge of Predicting the Unpredictable
}

\author{
Shuwei Zheng ${ }^{1}$, Jade Xiao Jue Soh ${ }^{1}$, Jiashen Loh $^{1}$, and Pushpalatha Bangalore \\ Lingegowda ${ }^{1}$ \\ ${ }^{1}$ Sengkang General Hospital
}

June 24, 2020

Letter to the Editor

\section{Introduction}

Since December 2019, severe acute respiratory syndrome coronavirus (SARS-CoV) 2 has caused an unprecedented public health crisis. Till date, more than two million cases have been diagnosed worldwide. In Singapore, where all confirmed cases are hospitalized early in the outbreak, immense pressure has been placed on the public healthcare system given the personal protective equipment and isolation needs in caring for these highly infectious patients. Coronavirus disease 2019 (COVID-19) transmission within healthcare facilities is a serious threat to medically vulnerable patients and healthcare workers. [1] We describe our experiences with regards to three atypical COVID-19 cases, seen in our institution.

\section{Case Reports}

Case 1 is a 67 -year-old man, with ischemic heart disease, diabetes mellitus, hypertension, dyslipidemia. He presented with a three-day history of dyspnea without preceding fever or respiratory complaints. He had low grade pyrexia of $37.8^{\circ} \mathrm{C}$ but unremarkable pulmonary auscultation. His initial chest radiograph was unremarkable (Figure 1A). Oropharyngeal swab for SARS-CoV-2 real-time polymerase chain reaction (RTPCR) returned positive. In the two weeks preceding his symptom onset, he had largely confined himself at home in compliance with nationwide measures to curb the ongoing outbreak. His only household contact was his wife, who had been asymptomatic. He developed fever of $38.7^{\circ} \mathrm{C}$ on his fourth hospitalization day, associated with clinical deterioration. Repeat chest radiograph showed interval development of bilateral midto-lower zone air space changes suggestive of pneumonia (Figure 1B). He was given lopinavir/ritonavir and his maximum oxygen requirement was $2 \mathrm{~L} / \mathrm{min}$ via nasal prongs during his ten-day hospitalization stay.

Case 2 is a 52-year-old female with poorly controlled diabetes mellitus, hypertension and dyslipidemia. She presented with acute onset left flank pain associated with dysuria, fever, chills and rigors, but no respiratory symptoms. Clinical examination on presentation revealed fever $\left(40.6^{\circ} \mathrm{C}\right.$ ), tachycardia (pulse rate 120 beats $/ \mathrm{min}$ ) and left costovertebral tenderness. Urine dipstick was positive for leucocytes and nitrites, with subsequent urine culture growing Escherichia coli . Procalcitonin was raised at $121.92 \mathrm{ug} / \mathrm{L}$. She was screened for SARS-CoV-2 RT-PCR via oropharyngeal swab in view of incidental right lower zone opacities seen on chest radiograph (Figure 1C), as part of our hospital's SARS-CoV-2 pneumonia surveillance program. Results were positive on two consecutive swabs, confirming the diagnosis of COVID-19. Left perinephric fat stranding (Figure 1D) was seen on abdominal computed tomography (CT) scan. Its basal lung views revealed patchy ground glass consolidation (Figure 1E). She improved clinically without need for supplemental oxygen. The diagnosis of COVID-19 would have been missed had she presented prior to chest radiographic changes or if no chest radiograph was performed. 
Case 3 is a 49-year-old man without known medical history. He presented with a five-day history of left breast lump, suggestive of an abscess. He had no preceding fever, myalgia, headache, respiratory symptoms, gastrointestinal symptoms, anosmia or dysgeusia. He was found to have newly diagnosed diabetes mellitus. Admission chest radiograph was unremarkable (Figure 1F). Bacterial culture of abscess aspirate grewStaphylococcus aureus . In view of an ongoing SARS-CoV-2 outbreak in the dormitory where this patient had been staying, he was screened for SARS-CoV-2 RT-PCR from an oropharyngeal swab, which returned positive.

\section{Discussion}

We illustrate three cases of atypical COVID-19. All patients were admitted into single room facilities, where they were screened positive. These patients could otherwise have been admitted to cohort cubicles where the propensity for secondary spread, would have been higher.

The 2003 SARS-CoV-1 outbreak in Singapore saw sequential spread involving three major acute care hospitals, the first arising following the hospitalization of a returning traveller with atypical pneumonia. Nosocomial transmission of SARS-CoV-1 resulted, taking a total of three weeks to control in the first hospital cluster. [2] The index patient central to another cluster of 60 patients at a second hospital in March 2003, had presented with atypical features, masked by E. coli bacteremia. [2-3] Our small case series illustrate the vulnerability of our inpatient cohort where atypical COVID-19 presentation can potentially result in secondary nosocomial transmission.

Contrary to the earlier SARS-CoV-1 outbreak, Singapore's first local case of SARS-CoV-2 was diagnosed promptly on January 23, 2020 in a traveller from Wuhan, China. He presented with typical febrile respiratory symptoms, and was diagnosed with pneumonia upon hospitalization. [4] Asymptomatic transmission of SARS-CoV-2 adds to the challenge of SARS-CoV-2 diagnosis and containment. Asymptomatic ratio of COVID-19 has been estimated to be as high as 30.8\% amongst Japanese nationals evacuated from Wuhan, China. [5] This is well-illustrated by our Case 3, where an ongoing outbreak in that setting, raises the pre-test probability of infection and underscores the immense challenge at curtailing transmission.

A high index of suspicion, strict infection control and prompt isolation measures are crucial in protecting our healthcare system from nosocomial SARS-CoV-2 outbreaks.

\section{Funding:}

Nil

\section{Conflict of Interest}

Nil

\section{References}

[1] Epidemiology of COVID-19 in a Long-Term Care Facility in King County, Washington. N Engl J Med. 2020 Mar 27. [Epub ahead of print]

[2] Gopalakrishna G, Choo P, Leo YS, Tay BK, Lim YT, Khan AS, Tan CC. SARS Transmission and Hospital Containment. Emerg Infect Dis. 2004 Mar;10(3):395-400.

[3] Tan TT, Tan BH, Kurup A, Oon LLE, Heng D, Thoe SYS, Bai XL, Chan KP, Ling AE. Atypical SARS and Escherichia coli Bacteremia. Emerg Infect Dis. 2004 Feb;10(2):349-352.

[4] Ministry of Health, Singapore. Confirmed Imported Case of Novel Coronavirus Infection in Singapore; Multi-Ministry Taskforce Ramps Up Precautionary Measures. 2020 Jan 23. https://www.moh.gov.sg/newshighlights/details/confirmed-imported-case-of-novel-coronavirus-infection-in-singapore-multi-ministrytaskforce-ramps-up-precautionary-measures

[5] Nishiura H, Kobayashi T, Suzuki A, Jung SM, Hayashi K, Kinoshita R, Yang Y, Yuan B, Akhmetzhanov 
AR, Linton NM, Miyama T. Estimation of the asymptomatic ratio of novel coronavirus infections (COVID19). Int J Infect Dis. 2020 Mar 13;S1201-9712(20)30139-9.

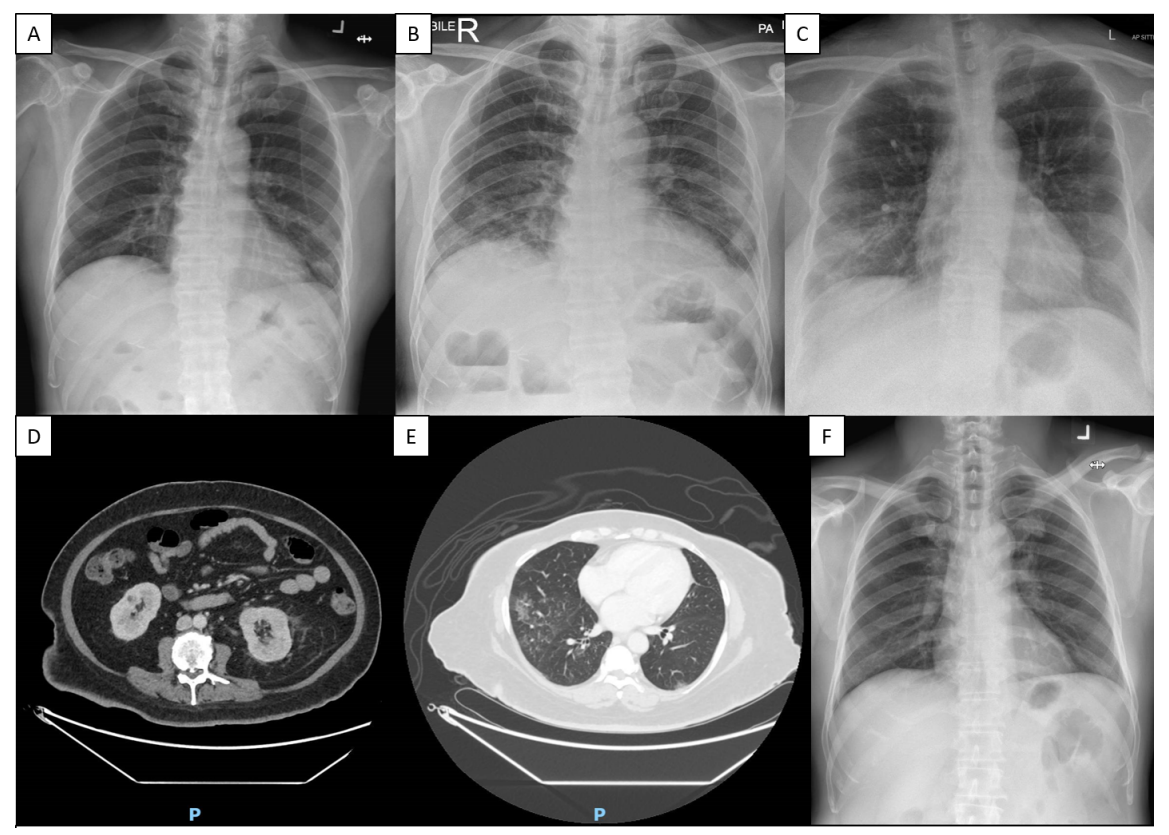

